\title{
What if your inversion has no numerical target?
}

\author{
Chris Wijns* \\ Dept. of Geology and Geophysics \\ University of WA, Australia \\ cwijns@geol.uwa.edu.au
}

\author{
Fabio Boschetti \\ CSIRO Exploration and Mining \\ Australia \\ fabio.boschetti@csiro.au
}

\author{
Peter Kowalczyk \\ Placer Dome Inc. \\ Canada \\ peter_kowalczyk@placerdome.com
}

\section{SUMMARY}

We present a system for inverting geological models in cases where there are no established numerical criteria to act as inversion targets. The method of interactive evolutionary computation provides for the inclusion of qualitative geological expertise within a rigorous mathematical inversion scheme, by simply asking an expert user to visually evaluate a sequence of model outputs. The traditional numerical misfit is replaced by a human appraisal of misfit. A genetic algorithm provides optimal convergence into the target parameter space, while optimising an ensemble of solutions, so that the non-uniqueness of the problem may be explored. In order to facilitate analysis of the results, we employ a visualisation technique known as self-organised mapping to represent the parameter space covered by the numerous model outputs. The result is a simple view of an otherwise complicated multi-dimensional problem. A user may infer much about the controlling parameters in the model through a few graphical displays of the data.

The potential of this interactive inversion and visualisation technique is demonstrated when we invert a geodynamic model for a conceptual pattern of fault spacing during crustal extension. We also present an example where the interactive scheme is linked to a numerical inversion of induced polarisation data. In this case, we are exploring for the numerical inversion parameters which lead to a particular geological output.

\section{INTRODUCTION}

Although it is desirable to have a quantitative measure of misfit for inversions, we are researching areas where visual judgment is necessary to evaluate model results in the absence of sufficient constraints. In many applications of geodynamic modelling, a forward solution is judged visually according to its resemblance to patterns seen in the field, to the fact that it does not contradict basic geological principles, or simply according to the modeller's expectations.

If we accept the fact that some inversion targets may be difficult to quantify, then we need a method to incorporate human interaction in directing the inversion process. Recently, research in artificial intelligence has resulted in systems to support such human interaction in optimisation problems (Takagi, 2001). They have been used in such diverse fields as graphic design, music composition, and the engineering of hearing aids. These systems are known collectively under the term interactive evolu- tionary computation (IEC).

We have extended the use of IEC to geological applications in which we are targeting model results for which we have no appropriate quantitative measure. The system represents an advance on time-consuming trial and error approaches by providing a formal role in the inversion process for geological experience which cannot be transformed into data. The traditional numerical measure of data mismatch is replaced by the user's subjective evaluation. Interactive inversion works by producing different solutions and presenting them to the user for judgment and ranking. Our system ultimately provides a range of solutions, which allows an assessment to be made about the nonuniqueness of the problem.

We have adopted a technique known as self-organised mapping (Kohonen, 2001) as a means to effectively view the range of inversion results. This visualisation method creates an easy environment in which to draw conclusions regarding parameter controls on the model. The roles of individual parameters in contributing to the non-uniqueness of the solution can be gauged through a few simple plots.

Although numerical inversions, in principle, do not need any visual evaluation, many available software programs return a single result, depending upon chosen inversion parameters such as misfit tolerance, smoothness criteria, etc. In this case, our interactive inversion is used to explore the influence of the inversion parameters, and not the geological parameters, on a desired geological outcome. The IEC method, together with the visualisation of the results, provides a systematic way to evaluate the roles of the inversion parameters in creating a class of geologically meaningful results.

For a geodynamic application, we have chosen to investigate the rheological controls on widely spaced faulting in a section of the Earth's crust which is undergoing extension. The coupled interactive and numerical application involves inverting chargeability data from an induced polarisation (IP) survey over a veinhosted sulphide system.

\section{METHOD}

The interactive inversion proceeds as follows: a user runs a modelling code with the aim of producing a geological model that matches a conceptual target. A number of selected parameters is allowed to vary within given ranges. The system initially generates a suite of different model outputs using randomly chosen parameter values. In the two examples below, the model outputs are either forward model images of a faulted crust, or 2D sections of numerically inverted chargeability data. 
Since we have no automated method for discriminating between geologically appropriate results, the user ranks each of them according to criteria founded in his or her experience and knowledge. A relative target misfit is now contained within these rankings, and the rankings are provided to a genetic algorithm (GA). The GA modifies the solution set by applying parameter swapping between highly-ranked models, generating new sets of models that progressively converge towards the target. A general overview of GAs is provided by Goldberg (1989), and details specific to our inversion method can be found in Boschetti et al. (1996). As in biological evolution, an element of randomness exists in the generation of new models, so that unexpected results may suggest new possibilities outside the experience or expectation of the geologist.

At the end of the interactive inversion, a target has been reached, and the best result is associated with a particular combination of the variable parameters. However, a number of model results may satisfy the target, or come close to it, so that we have an opportunity to investigate the non-uniqueness of the parameter values which all result in similar outputs. For this we use a self-organised map (SOM), which is a transformation of highdimensional $(n \mathrm{D})$ data into a lower-dimensional (usually 2D) plot. It is a classification algorithm which separates all the input data into clusters according to similarity. Topology is preserved, i.e. two points lying close to one another in the higher dimensional space also do so in the $2 \mathrm{D}$ space.

The 2D SOM which we use is a display composed of two different types of nodes: data nodes and distance nodes. The data nodes represent the $n \mathrm{D}$ data points, which are composed of the set of variable model parameters in the interactive inversion. Adjacent data nodes reflect points in $n \mathrm{D}$ space which are similar. The distance nodes connect the data nodes, and give an indication of the relative difference between the $n \mathrm{D}$ points. This may be measured, for example, as the euclidean distance between points. Thus, the input data vectors are assigned to particular data nodes, and the connecting distance nodes are coloured to show the magnitude of the distance. Data nodes are also shaded, according to an average of surrounding distance nodes, to produce a more continuous map. Clusters of data nodes close together in the SOM represent collections of parameter vectors which produce similar model results. The SOM plots in this work have been obtained with the use of the Matlab ${ }^{\mathrm{TM}}$ SOM Toolbox, written by Juha Vesanto.

\section{APPLICATIONS}

\section{Geodynamic Modelling}

The geodynamic example of faulting during crustal extension uses a particle-in-cell finite element code which is well-suited to problems involving very large deformation (Moresi, 1999; Moresi et al., 2002). The goal is to find sets of material parameters which give rise to fault spacing wider than the thickness of the upper crust. The location of faults is extremely sensitive to initial perturbations, both in nature and in our numerical models, so that a specific target image is not appropriate: we look for general behaviour and relative spacing. Our numerical model is composed of two initially homogeneous layers, a brittle upper crust overlying a ductile lower crust. The model is extended from one end and faults develop naturally through strain localisation.

Six model parameters are allowed to vary: the viscosity $\eta$ of the upper crust, and five yield parameters which describe the brittle failure of the upper crust. These are the cohesion $B_{o}$, the pressure dependence $B_{p}$ of the maximum sustainable shear stress, which is equivalent to the friction coefficient in Byerlee's Law (Byerlee, 1968), the tensile limit $B_{c}$ of the crustal rocks, the maximum proportion of strain weakening $E_{a}$, and the "saturation strain" $E_{o}$ beyond which no further weakening takes place.

Six generations of models are run, with eight simulations per generation. A user ranks the output images of each generation, from 1 to 8 , according to their similarity with the conceptual target of widely-spaced faults. Successive rankings of the outputs of each generation allow the algorithm to progressively hone in on combinations of parameter values which produce images approximating the target increasingly well. Figure 1 shows the progression of the simulations and the rankings accorded to each output image, for the first, second, and sixth (last) generation of the inversion process. Dark areas in the upper crust denote plastic strain, sometimes localised into welldefined shear bands, which are our representation of faults. A number of models are not extended because they fail to converge numerically. These are left unranked, and the GA ranks them randomly at the bottom of the suite of solutions and does not explore further in this area. Later analysis reveals that an unrealistically high yield strength is the reason for the numerical failing. Thus the loss of this particular area of parameter space does not equal a loss of physically plausible solutions. Although the first generation (panel i) does not produce anything resembling the target image, the algorithm still eventually finds, through the continued high ranking of the best results, a solution which we deem acceptable. At each generation, model results are compared with the best-ranked solution of the previous generation in order to assess whether we are converging towards the target. By generation 6 , half of the models exhibit qualitatively similar solutions, and we halt the process.

Figure 2 illustrates the mapping of the output images onto the SOM. All extension images have been labelled with their "absolute" rank at the end of the entire inversion exercise, 1 being the best model. This is different from the relative ranking performed during the inversion run. SOM nodes which are close together in Figure 2, and separated by small distances, represent clusters of input vectors which result in similar output images. It is clear that the area in parameter space at the top left corner contains the models that are closest to the target. At this point one may observe that some models far from the "best" corner (e.g. the model ranked 5.5 in Figure 2) nevertheless share characteristics of the best models. This is indicative of the nonuniqueness of inversion solutions - models with very different parameter inputs may produce nearly identical outputs.

The actual parameter values can be displayed in various derivatives of the SOM plot which are not shown here. We can analyse the influence of each model parameter on the solution. A high viscosity is essential for producing the best model, together with relatively small values for the other five components, includ- 


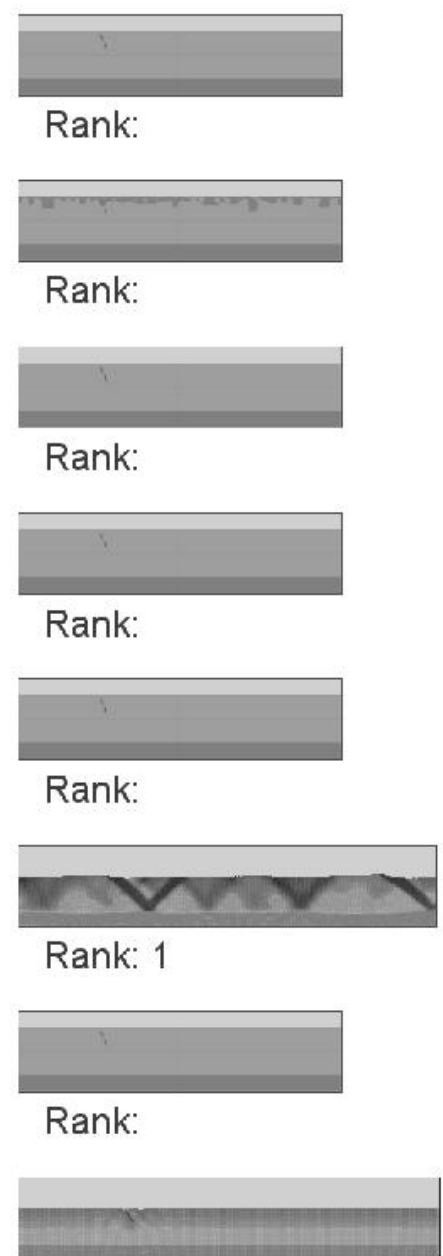

Rank: 2

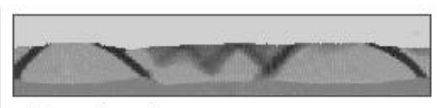

Rank: 1

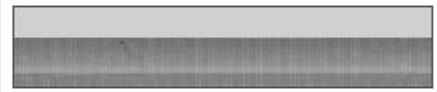

Rank: 5

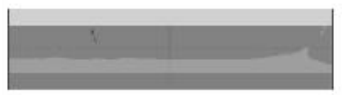

Rank:

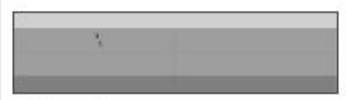

Rank:

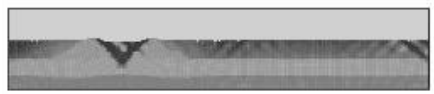

Rank: 3

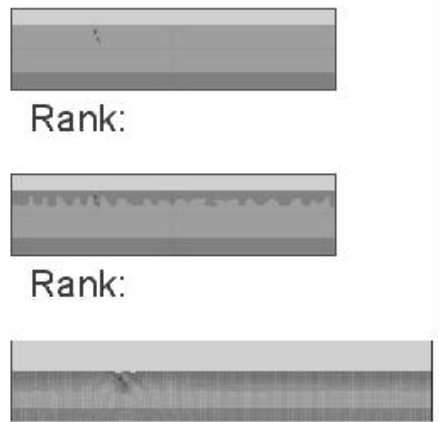

Rank: 4

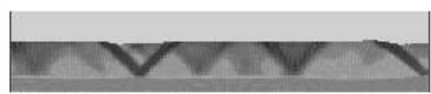

Rank: 2 (best of gen. 1)

ii

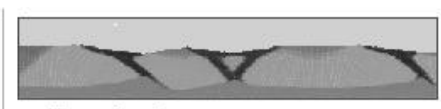

Rank: 2

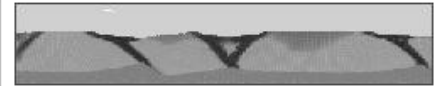

Rank: 5

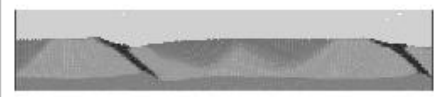

Rank: 1

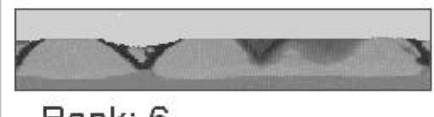

Rank: 6

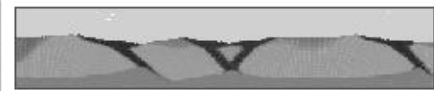

Rank: 4

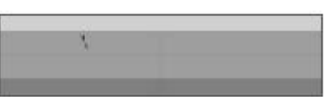

Rank: 9

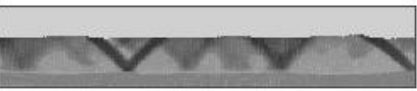

Rank: 7

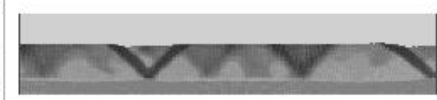

Rank: 8

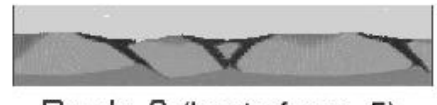

Rank: 3 (best of gen. 5)

iii

Figure 1: Evolution of the interactive inversion. Panels (i) to (iii) represent the first two and the last (sixth) generation of the algorithm. Images are ranked according to their fault spacing, where dark areas in the upper crust indicate accumulated plastic strain (faults). Some models have not been extended to full length because of numerical non-convergence. These are left unranked, and the algorithm orders them randomly so as to fill up the bottom rankings. The third image of generation six (panel iii) is the best result.

ing the ratio $E_{a}$ of strained to intact rock strength, denoting weak faults. The last component, $E_{o}$, can in fact vary somewhat without affecting the final result, which is indicative of the non-uniqueness of the solution with respect to this parameter.

\section{Numerically Inverted IP Data}

Numerical inversions do not require user interaction, but often return a single solution. In the following example, we explore the effects, on the geological picture, of changing the numerical inversion parameters for chargeability data from a dipoledipole IP survey. The IEC scheme is coupled to the University of British Columbia IPINV2D code. The variable parameters are the data misfit tolerance, closeness to the reference model, and vertical and horizontal smoothness. The geological target is a vein-hosted sulphide system, so the model outputs that show compact discrete zones are the most plausible. The data were collected many years ago, and subsequently digitised from paper pseudo-sections, so that there is no record of measurement errors, noise, etc. The appropriate misfit tolerance, for example, would only be found by exploratory data analysis. Interactive inversion is an organized way to investigate the parameters.

Figure 3 shows the third generation of the IEC process. Each image is the result of a single IP inversion. The depth of inves- 


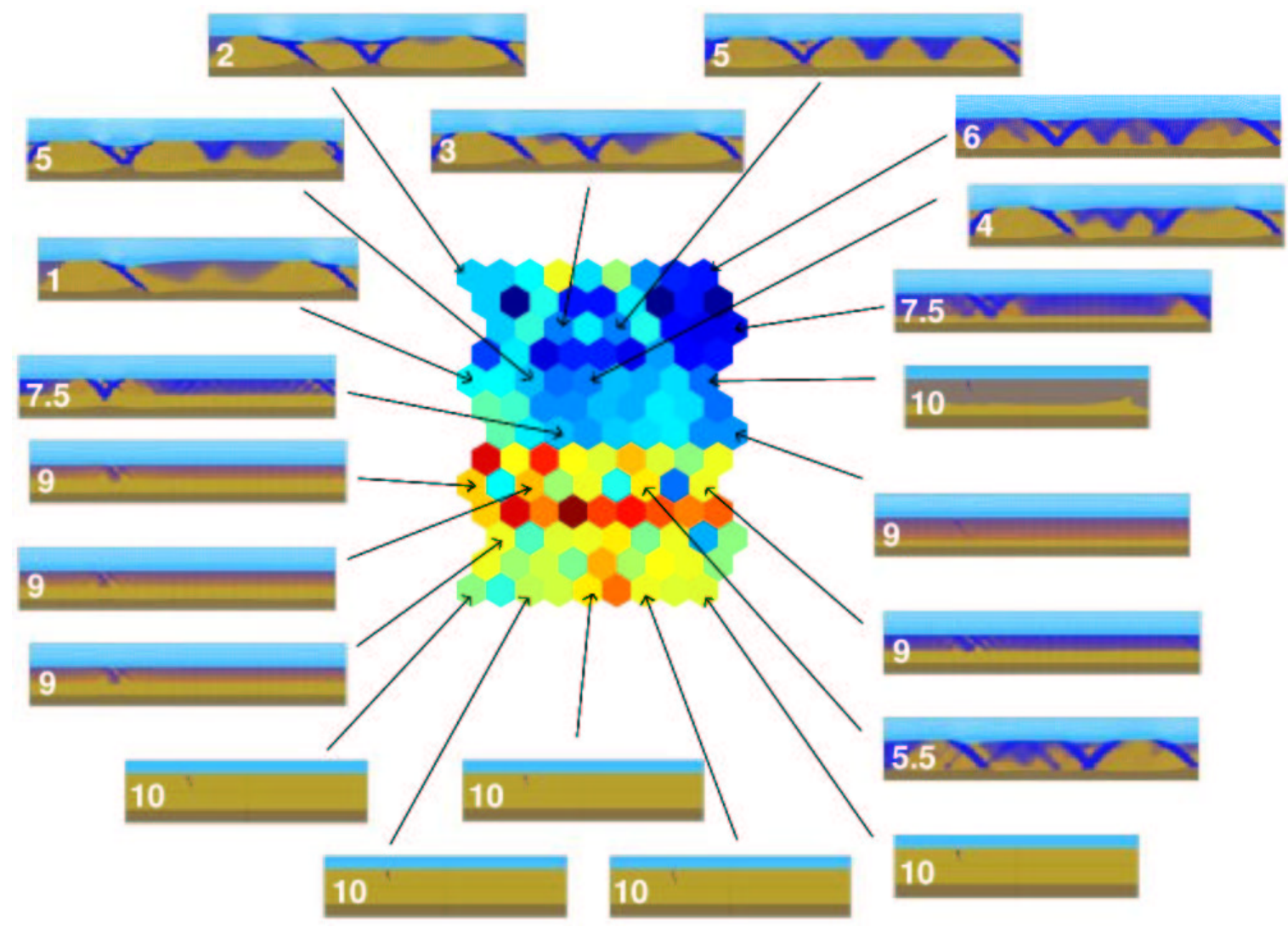

Figure 2: Mapping of output images to SOM nodes. Data nodes (every second node) represent outputs from the inversion process. The output images are labelled with their absolute rankings. The colours of the distance nodes in the SOM vary from blue (close) to red (far). Data nodes are coloured according to an average of surrounding distance nodes. The best model images are clustered in the top left domain.

tigation is not large, so the inverted results can be interpreted to be showing the tops of veins. The first two generations had high rankings assigned to images showing discrete zones of chargeability. In the third generation, we have five models which match this conceptual target, although they are the result of different numerical inversion parameters. The SOM analysis is not shown.

\section{DISCUSSION AND CONCLUSION}

In the above examples, we are looking for model results which we are not able to sufficiently describe by numerical measures. Finding a suitable combination of parameters which gives rise to the target would previously have involved one of two more laborious approaches: the manual selection of parameters by trial and error, or an exhaustive coverage of all parametric space. Trial and error may succeed with a limited number of parameters, but depends upon the user's knowledge of the coupling and feedback between them, which, in highly non-linear problems such as the example involving crustal rheologies, may be impossible. A parametric sweep quickly becomes unfeasible due to the sheer number of models which must be run as the number of parameters is increased. In the faulting example, in excess of 20000 models would have to be run in order to cover all possible parameter combinations, and each forward model takes a few hours to run on a $935 \mathrm{MHz}$ desktop computer with $500 \mathrm{MB}$ of RAM. Using IEC, we have found a number of solutions with only 48 models being run. This vast reduction in individual models can be attributed to the fact that visual ranking provides more information in this type of search than numerical misfit in a non-interactive inversion. A model containing one or more features of paramount importance, but with a potentially large numerical misfit because of, for example, spatial discrepancy in feature locations, is ranked highly and provides a significant step forward in the search through parameter space. In fact, because of the combinatorial nature of the GA progression, two images which each contain a different feature of importance can both be ranked highly in order to increase the likelyhood of producing a new model containing both desired features. In both the geodynamic modelling case, and the coupled numerical and interactive inversion, neither trial and error nor a parametric sweep takes full advantage of the knowledge of a user.

The power of inversion lies in demonstrating the range of nonuniqueness of a solution, and using a SOM, we perform an a posteriori investigation of the sensitivity of the results to changes in our chosen variables. The GA optimises an ensemble of solutions, which provides the range of non-unique results, and the SOM represents the multidimensional parameter space in a clear and simple 2D visualisation environment. It is easy to 


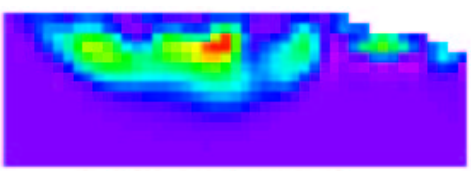

disseminated

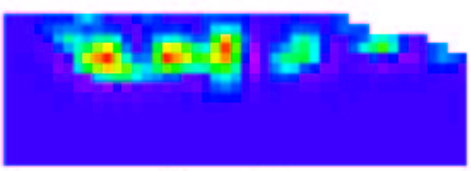

discrete

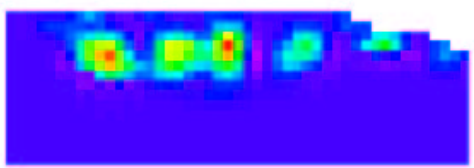

discrete

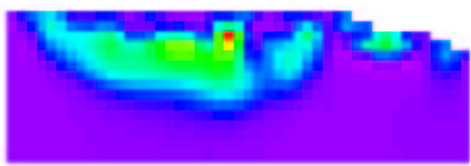

disseminated

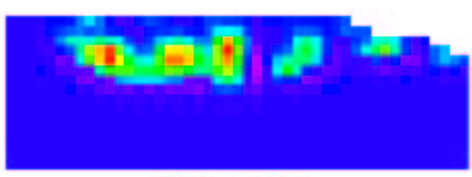

discrete

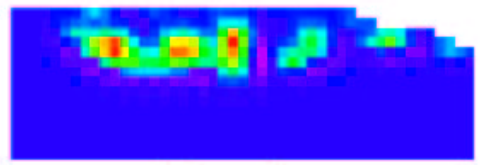

discrete
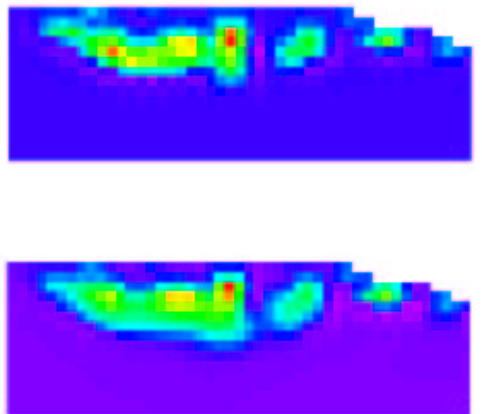

disseminated

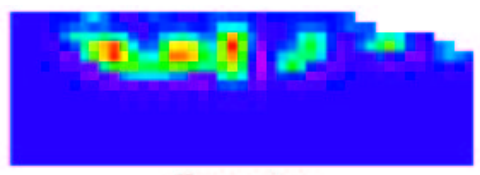

discrete

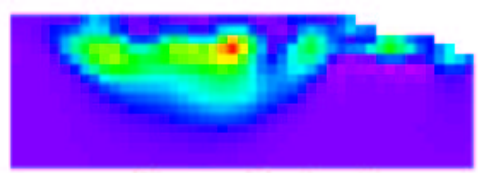

disseminated
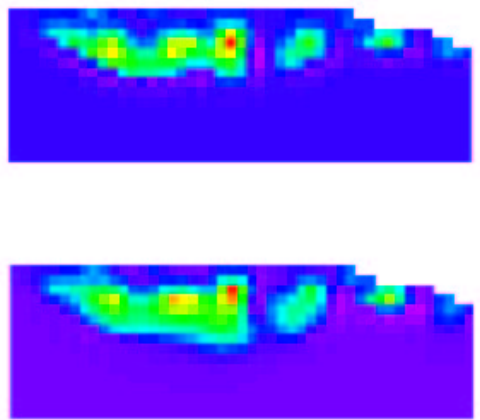

disseminated

Figure 3: Third generation of IP inversions. Five out of twelve images show discrete zones of chargeability, but are the result of different numerical inversion parameters.

draw conclusions regarding the controlling factors in the model, and the connections between them. The IEC system and SOM visualisation can be applied to any modelling problem which would benefit from human interaction.

\section{References}

Bellingham, P., White, N., 2000. A general inverse method for modelling extensional sedimentary basins. Basin Research 12, 219-226.

Boschetti, F., Dentith, M., List, R., 1996. Inversion of seismic refraction data using genetic algorithms. Geophysics 61 (6), $1715-1727$.

Byerlee, J. D., 1968. Brittle-ductile transition in rocks. Journal of Geophysical Research 73, 4741-4750.

Cross, T., Lessenger, M., 1999. Construction and application of a stratigraphic inverse model. In: Harbaugh, J., Watney, W., Rankey, E., Slingerland, R., Goldstein, R., Franseen, E. (Eds.), SEPM Special Publication 62: Numerical Experiments in Stratigraphy: Recent Advances in Stratigraphic and Sedimentologic Computer Simulations. SEPM (Society for Sedimentary Geology), pp. 69-83.

Goldberg, D., 1989. Genetic Algorithms in Search, Optimization, and Machine Learning. Addison-Wesley, Reading, Massachusetts, $412 \mathrm{pp}$.
Kohonen, T., 2001, Self-organizing maps: 3rd Edition, New York, Springer, Series in Information Sciences, v. 30, 501 p.

Moresi, L., Aug. 1999. Ellipsis, http://www.ned.dem.csiro.au/research/solidMech/PIC.

Moresi, L., Dufour, F., Mühlhaus, H.-B., 2002. Mantle convection models with viscoelastic/brittle lithosphere: Numerical methodology and plate tectonic modeling. Pure and Applied Geophysics 159, 2335-2356.

Takagi, H., 2001. Interactive evolutionary computation: Fusion of the capacities of EC optimization and human evaluation. Proceedings of the IEEE 89 (9), 1275-1296.

Tarantola, A., 1987. Inverse Problem Theory. Elsevier, Amsterdam, $613 \mathrm{pp}$. 